\title{
Impact of help-seeking behavior and partner support on postpartum depression among Saudi women
}

\author{
Adel F Almutairi ${ }^{1,2}$ \\ Mahmoud Salam ${ }^{1,2}$ \\ Samiyah Alanazi' \\ Manal Alweldawi' \\ Najad Alsomali' \\ Najla Alotaibi' \\ 'King Saud Bin Abdulaziz University \\ of Health Sciences, ${ }^{2}$ Science and \\ Technology Unit, King Abdullah \\ International Medical Research \\ Center, Ministry of National Guard \\ Health Affairs, Riyadh, Saudi Arabia
}

\author{
This article was published in the following Dove Press journal: \\ Neuropsychiatric Disease and Treatment \\ 20 July 2017 \\ Number of times this article has been viewed
}

Background: Many studies have discovered a number of factors that can contribute to the risk of developing postpartum depression (PPD), including, but not limited to, life stressors, lack of social support, low economic status, and quality of the marital relationship. However, these studies were conducted in various countries with participants from different cultural backgrounds.

Purpose: This study aimed to examine the impact of general help-seeking behavior (GHSB) and partner support (PS) on PPD among Saudi women in primary health care clinics in Riyadh city. Methods: Data were collected by using self-administered measures of the Edinburgh Postnatal Depression Scale (EPDS), General Help-Seeking Questionnaire (GHSQ), and Partner Support Scale (PSS). Frequency distribution was used to analyze the categorical data, and Student's $t$-test and one-way analysis of variance were employed to compare the numerical data. Linear regression analysis was used to control for all confounders.

Results: The findings showed that $9 \%$ and $28 \%$ of women had good and poor GHSB, respectively, $16 \%$ had poor PS, and $25.7 \%$ could be classified as probably depressed. Negative relationships between GHSB versus PPD and PS versus PPD were observed. Adjusting by mode of delivery and controlling for confounders in linear regression showed that women who underwent normal vaginal delivery, with higher para rates $(\beta=0.250, t=2.063)$ and lower PS scores $(\beta=-0.238, t=-2.038)$, were more likely to suffer higher depression scores ( $\operatorname{adj} P=0.043$ and adj $P=0.045$, respectively). Women who underwent cesarean-section, with postpartum duration $\geq 6$ weeks $(\beta=0.374, t=2.082$ ), were more likely to suffer higher depression scores (adj $P=0.045$ ) compared to those with $<6$ weeks of postpartum duration.

Conclusion: The prevalence of PPD among the study participants was high, especially among higher para women who underwent normal delivery and women $\geq 6$ weeks post cesareansection, in comparison with the results in other studies. PPD is reduced by enhancing women's GHSB and PS.

Keywords: postpartum depression, partner support, help-seeking, women, Saudi Arabia

\section{Introduction}

Postpartum depression (PPD) is a mental disorder that can affect women and their families if not properly diagnosed and treated. It is a major health problem that can start as early as the delivery day and lasts up to 1 year. PPD can vary from mild to severe, affecting all women regardless of the mode of delivery, para, and other social stressors such as income, age, race, culture, and education. Symptoms may include fatigue, mood swings, excessive crying, isolation, panic, and irritability that can interfere with the mothers' daily activities. It is noteworthy that PPD is different from
Correspondence: Adel F Almutairi King Abdullah International Medical Research Center (KAIMRC), Ministry of National Guard-Health Affairs, Riyadh II 426, Saudi Arabia

Tel +966555900336

Email almutairiad I@ngha.med.sa 
postpartum blues and psychosis, in which the first refers to a transient condition mood liability, mild anxiety, and tearfulness that most mothers could experience shortly after delivery. Whereas postpartum psychosis is a rare illness that occurs in $\sim 1-2$ women out of 1,000 and is considered to be the most severe form of postpartum psychiatric illnesses. ${ }^{1}$

The consequences of PPD make it a serious public health concern as it could negatively impact the development of maternal confidence as well as the cognitive, emotional, and social development of the infant. ${ }^{2,3}$ According to the World Health Organization's (2009) report, PPD is prevalent in both developed and developing countries to varying degrees: $\sim 10 \%-15 \%$ in developed countries and 20\%-40\% in developing countries. ${ }^{4}$ Interestingly, it is more prevalent among young mothers and single mothers. The causes of PPD remain unknown; however, many factors can mitigate and increase its risk, including psychological, biological, social, and cultural factors. For instance, a meta-analysis of 84 studies that was conducted by Beck indicated that life stressors, lack of social support, low self-esteem, prenatal depression, low economic status, quality of the marital relationship, and unwanted pregnancy are risk factors for PPD. ${ }^{5}$ Many studies conducted in different settings showed a significant relationship between PPD and the quality of the mother's relationship with her partner. ${ }^{6-8}$ Thus, assessing the partner's perceived support can help determine to what degree the partner contributes to alleviating stress and depression among postpartum women.

Although PPD and its risk predictors among postpartum mothers have been assessed in various communities with participants from different cultural backgrounds, few have investigated the impact of general help-seeking behavior (GHSB) and partner support (PS) on PPD. In general, when people are psychologically distressed, there is a tendency for them to seek informal help from their friends and family members, rather than from a medical professional or psychologist. ${ }^{9}$ One study recommended that the concept of informal help-seeking itself should be promoted, rather than just restricting it to professional sources. ${ }^{10}$ However, there is an insufficient number of studies that examine the effect of GHSB and PS on PPD in communities with distinctive characteristics, such as Saudi Arabia. The Saudi Arabian community has unique cultural features that are deeply religious, conservative, traditional, and collectivistic, meaning that every family member is responsible for the others. It is also strongly influenced by Islamic values that encourage supporting, visiting, respecting, and helping family members. The purpose of this study was to evaluate the impact of GHSB and PS on PPD among Saudi women in primary health care (PHC) clinics in Riyadh city. Specifically, we aimed to describe some pregnancy-related characteristics; explore the relationship between GHSB, PS, and depression, as perceived by the study participants; and determine the factors associated with higher PPD scores.

\section{Methods}

\section{Study design}

This was a cross-sectional study, using a number of validated questionnaires.

\section{Study setting}

The Ministry of National Guard-Health Affairs provides health care services to the national guards, their dependants, and employees. In the central region of Riyadh, the ministry's PHC facilities are distributed across four main centers serving a population of 180,000 . On average, a patient visits a $\mathrm{PHC}$ physician approximately four times per year. The number of patients examined by PHC physicians on a daily basis ranges from 12 to 15 , with $150 \mathrm{PHC}$ physicians serving a population of 60,000. Two of these polyclinics are Khashmalaan and Iskan clinics located in the eastern quarter of Riyadh.

\section{Population and sampling}

The two polyclinics were targeted because when postpartum women are discharged from the in-hospital setting, they are scheduled for regular follow-up visits at these sites. Using the convenience sampling approach, women who came for a routine postpartum follow-up visit were surveyed. Those who were undergoing treatment for psychological problems or mothers whose babies were diagnosed with serious health problems, were stillborn, or experienced intrauterine fetal death were excluded. With a $13 \%$ prevalence of PPD as reported by a meta-analysis paper, as well as based on the results of a large number of studies, ${ }^{11}$ a 0.06 margin of error, and a 95\% confidence level, the required sample size was calculated to be 120 .

\section{Data collection}

The data collection team was composed of midwifery students from the College of Nursing, King Saud Bin Abdulaziz University of Health Sciences, Riyadh. This team was trained by the principal investigator on how to introduce the study objectives to the participants in the targeted setting, present them with the survey forms, and collect their responses. If the study participant was illiterate, the midwife conducted the survey through a face-to-face interview. 
Three questionnaires were used to collect the data in this study, namely the Edinburgh Postnatal Depression Scale (EPDS), General Help-Seeking Questionnaire (GHSQ), and Partner Support Scale (PSS). The EPDS is a self-reporting tool originally developed at health centers in Scotland. It has been widely employed and validated in many countries and different contexts. It is one of the most commonly used scales to identify mothers who are potentially suffering from postnatal depression. It is composed of 10 items to assess the mother's emotional experience over the past 7 days. Mothers who scored $\geq 13$ on the EPDS were considered to be possible PPD sufferers and those who scored $<13$ were considered normal.

Although many studies reported good validity and reliability of the Edinburgh tool in different contexts, ${ }^{12}$ the cutoff point (depressed/not depressed) is widely debated in the literature. For example, the cutoff point was initially established at nine in Guedeney and Fermanian's study. ${ }^{12}$ In another study conducted in Iran by Montazeri et al, three interpretive categories were created for the scores: those with a score of $\geq 13$ were to be considered postnatally depressed, while scores of 10-12 represented "borderline" and 0-9 "not depressed." ${ }^{13}$ However, Al-Modayfer et a ${ }^{14}$ in Saudi Arabia reported that the optimal cutoff point was 13 among Saudi women, with a sensitivity of 40.6 and specificity of 92.7 . Importantly, a recent systematic review by Shrestha et al ${ }^{15}$ noted that the potential of EPDS to identify depression among women from low- and lower-middle-income countries with different cultures is unclear. They concluded that 12 out of 14 studies that utilized the EPDS tool in non-English local language versions had lower precision for identifying true cases of depression among postpartum women compared to the studies using tools in the original English version. This led us to conduct the analytical statistical analysis using the depression score (continuous variable) as a more reflective and flexible description of the outcome measure, rather than the rigid and debatable cutoff point (categories).

The GHSQ was developed by Wilson et al. ${ }^{9}$ It assesses the intention of the target group to seek help from different sources, including formal and informal sources, for a certain problem, which is PPD in this study. This scale is rated on a 7-point Likert scale, ranging from (1) extremely unlikely to (7) extremely likely. This tool has been used in different studies with adequate reliability and validity. ${ }^{9}$ The last part of the current study's questionnaire contains the PSS, which is a self-administered scale composed of eight items that assess emotional support, financial support, dependability, and assistance with childcare. It is rated on a 4-point Likert scale where (1) indicates strongly disagree and (4) strongly agree. The theoretical range of this scale is $8-32$, where a high score indicates greater support and a low score indicates less support. The internal consistency of this scale was assessed using Cronbach's $\alpha(0.95)$, which indicated adequate reliability. ${ }^{16}$

The scores obtained from the three tools were summated individually, converted to percentage mean scores (PMSs), and then categorized. Women who reported an EPDS PMS $\geq 43 \%$ were considered depressed. The GHS behavior was poor (PMS $<40 \%$ ), average (PMS 40\%-75\%), or good (PMS $>75 \%$ ). The PS was either high (PMS $>50 \%$ ) or low (PMS $\leq 50 \%)$.

\section{Ethical considerations}

Ethical approval to conduct this study was obtained from the Institutional Review Board Committee at Ministry of National Guard-Health Affairs, Saudi Arabia. Participation was completely voluntary, and no subject identifying data were obtained. The survey was conducted only after the purpose, procedure, benefits, and potential risks were explained to the respondents, and they consented by checking the "agree to participate" box on the survey cover letter. Since the survey was self-reported and kept in sealed envelopes, no influential biases or stressors were created by the data collection team.

\section{Data analysis}

The Statistical Package for the Social Sciences (Version 23; SPSS $)^{17}$ was used for data analysis. Categorical data of sample characteristics and responses were illustrated in frequencies and percentages. The assumption of normality was tested, which indicated that data were normally distributed. Accordingly, the arithmetic mean was used as a summary statistic for quantitative data. Standard deviation was used as a measure of dispersion. Student's $t$-test (two groups) and one-way analysis of variance (ANOVA) ( $\geq 3$ groups) were used as a test of significance to compare the numerical data. Two linear regression analyses, adjusted by mode of delivery, were used to control for all confounders (age, para, postpartum duration, GHSB score, and PS score) and to determine the significant factors associated with higher or lower PPD scores. For all statistical analyses, a $P$-value $<0.05$ was considered significant.

\section{Results \\ Sample characteristics}

Of the 130 questionnaires, 113 were completed by the female participants, all of whom were Saudi nationals, yielding 
a response rate of $87 \%$. Most of the women in this study were from 26 to 30 years of age $(n=38,36.6 \%)$; the age range of the second largest group $(n=30,26.5 \%)$ was from 31 to 35 years, and the third largest group $(n=24,21.2 \%)$ was in the range of 18-25 years. Approximately $66 \%$ of the surveyed women had a spontaneous vaginal delivery, while 34\% underwent a cesarean-section (C-section) delivery. Nearly half of the women were Para $1-2(n=55$, 48.7\%), 26.5\% ( $n=30)$ were Para 3-4, and 24.8\% $(n=28)$ were Para $\geq 5$. Of the women, $67 \%(n=76)$ were 6 weeks postpartum, while the rest ranged from 1 to 5 weeks postpartum. Most of the participants had a university level education $(n=57,50.5 \%), 39.8 \%(n=45)$ were school level graduates, and $9.7 \%(n=11)$ were uneducated (illiterate). Most of the participants were living in the capital city Riyadh (90\%), while $10 \%$ were living in proximity to the city. The participants' demographics are presented in Table 1.

\section{Evaluating the PPD, patterns of help- seeking behavior, and PS}

The overall PMS computed from the 10 statements of the EPDS was $31.0 \pm 17.8$, with $25.7 \%$ of women being probably

Table I Description of the study's sample characteristics

\begin{tabular}{ll}
\hline Variables & $\mathbf{N}=\mathbf{I}$ I 3 ( I 00\%) \\
\hline Age category (years) & \\
I8-25 & $24(21.2)$ \\
$26-30$ & $38(33.6)$ \\
3 I-35 & $30(26.5)$ \\
$\geq 36$ & 2 I $(18.7)$ \\
Duration since delivery & \\
$<$ I week & $5(4.4)$ \\
I week & $4(3.5)$ \\
2 weeks & $8(7.1)$ \\
3 weeks & $4(3.5)$ \\
4 weeks & $7(6.2)$ \\
5 weeks & $9(8.0)$ \\
6 weeks & $76(67.3)$ \\
Mode of delivery & \\
Normal vaginal & $75(66.4)$ \\
C-section & $38(33.6)$ \\
Para & \\
I-2 & $55(48.7)$ \\
$3-4$ & $30(26.5)$ \\
$\geq 5$ & $28(24.8)$ \\
Education level & \\
Illiterate & 1 I (9.7) \\
School level & $45(39.8)$ \\
College level & $57(50.5)$ \\
Income & \\
5,000 & $15(13.3)$ \\
$5,000-10,000$ & $60(53.1)$ \\
\hline A 0000 & $38(33.6)$ \\
\hline
\end{tabular}

Abbreviation: C-section, cesarean section. depressed (PMS $\geq 43$ ). Of the 113 women, $15.2 \%$ responded negatively to the statement "Being able to laugh and see the funny sides of things," and $15.1 \%$ replied negatively to the statement "I have looked forward with enjoyment to things as much as I ever did." Approximately 51\% of surveyed women "blamed themselves unnecessarily when things went wrong," while 39\% were "anxious or worried for no good reason;" $\sim 25.3 \%$ "felt scared or panicky for no very good reason" and 44.3\% "felt overwhelmed;" and $\sim 35.4 \%$ were "unhappy that they had difficulty sleeping," and $18.6 \%$ reported being "sad or miserable." Some women $(14.2 \%)$ reported that they "have been so unhappy that they have been crying," and 4.5\% actually admitted that the thought of harming themselves had occurred to them.

The GHSB scale questioned the intention of women to seek help from various sources in the community. The overall PMS of help-seeking behavior was 50.9 \pm 18.2 , with $9 \%$ reporting good help-seeking behavior, $28 \%$ reporting poor behavior, and $63 \%$ neutral behavior. The findings also indicated that $89.4 \%$ of participants sought help from their partners when they had personal or emotional problems. In addition, the participants had different scores concerning their willingness to seek help from other sources, such as parents $(85 \%)$, friends $(60 \%)$, mental health professionals (42\%), and religious leaders (Shaikh) (46\%).

Regarding the PS scale, the overall PMS was 71.7 \pm 22.5 . Approximately $84 \%$ had a good perception of their supportive partners, whereas $16 \%$ had a poor perception; $92 \%$ of women viewed their partners as "someone they can count on for financial support if they need it," and 88\% reported that "they can talk with their partners about things that are important to them." Of the surveyed women, $86 \%$ noted that their partners were "affectionate toward them," and 75\% reported that their partners "helped them in caring for their children." Most of the women (81\%) described their partners as "someone who understands how they are feeling," and $76 \%$ reported that they tend "to talk with them and spend time with them." In general, $88 \%$ of women claimed that they could count on their partners, and $69 \%$ admitted that their partners "do things with them."

\section{Factors associated with depression}

An indirect relationship was observed between the PMS of the GHSB and depression ( $y=53.2-0.08 x, P=0.506)$ on one side and between the PMS of the PS and depression $(y=77.9-0.2 x$, $P=0.023$ ) on the other sude. Initial bivariate analysis showed no statistically significant association between most of the pregnancy-related characteristics and depression scores as 
Table 2 Percentage mean scores of the Edinburgh Postnatal Depression Scale (EPDS), General Help-Seeking Questionnaire (GHSQ), and Partner Support Scale (PSS) with demographic variables

\begin{tabular}{|c|c|c|c|}
\hline \multirow[t]{3}{*}{ Variables } & EPDS & GHSQ & PSS \\
\hline & $\%$ mean score \pm SD & $\%$ mean score \pm SD & $\%$ mean score \pm SD \\
\hline & $31.0 \pm 17.8$ & $50.9 \pm 18.2$ & $71.7 \pm 22.5$ \\
\hline \multicolumn{4}{|c|}{ Age category (years) } \\
\hline $18-25$ & $31.8 \pm 20.4$ & $55.8 \pm 19.0$ & $78.9 \pm 19.4$ \\
\hline $26-30$ & $30.5 \pm 16.5$ & $49.0 \pm 16.0$ & $69.3 \pm 21.2$ \\
\hline $31-35$ & $30.0 \pm 14.9$ & $49.4 \pm 17.6$ & $71.3 \pm 21.3$ \\
\hline \multirow[t]{2}{*}{$\geq 36$} & $32.2 \pm 21.8$ & $50.8 \pm 22.0$ & $68.3 \pm 28.8$ \\
\hline & $F=0.087, d f=3, P=0.967$ & $F=0.774, d f=3, P=0.5 \mathrm{II}$ & $F=1.155, d f=3, P=0.330$ \\
\hline \multicolumn{4}{|c|}{ Duration since delivery } \\
\hline$<6$ weeks & $30.2 \pm 18.2$ & $51.1 \pm 17.9$ & $70.4 \pm 22.4$ \\
\hline \multirow[t]{2}{*}{$\geq 6$ weeks } & $31.4 \pm 17.8$ & $50.5 \pm 19.1$ & $74.3 \pm 22.8$ \\
\hline & $t=-0.328, P=0.743$ & $t=0.170, P=0.865$ & $t=-0.869, P=0.387$ \\
\hline \multicolumn{4}{|l|}{ Mode of delivery } \\
\hline Normal vaginal & $31.2 \pm 18.9$ & $51.8 \pm 19.3$ & $70.9 \pm 21.9$ \\
\hline \multirow[t]{2}{*}{ C-section } & $30.4 \pm 15.9$ & $48.9 \pm 15.9$ & $73.2 \pm 23.9$ \\
\hline & $t=0.226, P=0.822$ & $t=0.779, P=0.437$ & $t=-0.524, P=0.602$ \\
\hline \multicolumn{4}{|l|}{ Para } \\
\hline $0-2$ & $31.2 \pm 19.2$ & $51.6 \pm 19.1$ & $75.6 \pm 23.8$ \\
\hline $3-4$ & $30.1 \pm 16.3$ & $46.9 \pm 16.1$ & $68.1 \pm 19.7$ \\
\hline \multirow[t]{2}{*}{$\geq 5$} & $31.5 \pm 17.3$ & $53.8 \pm 18.5$ & $67.9 \pm 22.3$ \\
\hline & $F=0.05 \mathrm{I}, d f=2, P=0.950$ & $F=I . I \mid 0, d f=2, P=0.333$ & $F=1.645, d f=2, P=0.198$ \\
\hline
\end{tabular}

Notes: $t$, Student's $t$-test; $F$, one-way ANOVA; $P$-value: statistically significant at $<0.05$.

Abbreviations: C-section, cesarean section; ANOVA, analysis of variance; SD, standard deviation; df, degree of freedom.

indicated in Table 2. However, after adjusting by the mode of delivery and controlling for all possible confounders in two linear regression models, women who underwent normal vaginal delivery with higher para rates $(\beta=0.250, t=2.063)$ and lower PS scores $(\beta=-0.238, t=-2.038)$ were more likely to suffer higher depression scores (adj $P=0.043$ and adj $P=0.045$, respectively). Women who underwent C-section, with postpartum duration $\geq 6$ weeks $(\beta=0.374, t=2.082$ ), were more likely to suffer higher depression scores (adj $P=0.045$ ) compared to those of women $<6$ weeks postpartum. Table 3 shows the significant factors associated with higher depression scores.

\section{Discussion and conclusion}

The prevalence of PPD in this study was relatively high $(25.7 \%)$ in comparison with other studies in the region that reported a prevalence of $17.8 \%{ }^{18}$ However, the findings in this study were congruent with the World Health Organization's (2009) report, in which PPD was prevalent in developing countries $(20 \%-40 \%)$. The consequences of PPD make it a serious public health concern as it could negatively impact the development of maternal confidence and the cognitive, emotional, and social development of the infant. ${ }^{19,20}$ Although depression is associated with a broad range of demographic, psychosocial, financial, and lifestyle variables ${ }^{21}$ researchers should focus on more controllable factors, which, if modified, could alleviate postpartum stress and decrease depression. Improving help-seeking behavior and perception toward the spouse as a supportive partner are both malleable factors

Table 3 Significant factors associated with higher depression scores adjusted by mode of delivery

\begin{tabular}{|c|c|c|}
\hline \multirow[t]{4}{*}{ Variables } & \multicolumn{2}{|c|}{$\begin{array}{l}\text { Edinburgh Postnatal Depression Scale } \\
\text { linear regression }\end{array}$} \\
\hline & $\begin{array}{l}\text { Normal vaginal } \\
\text { delivery }\end{array}$ & $\begin{array}{l}\text { C-section } \\
\text { delivery }\end{array}$ \\
\hline & $\beta(t)$ & $\beta(t)$ \\
\hline & Adj $P$-value & Adj $P$-value \\
\hline \multirow[t]{2}{*}{ Age $(>30$ vs $\leq 30)$} & $-0.068(-0.563)$ & $0.036(0.188)$ \\
\hline & Adj $P=0.575$ & Adj $P=0.852$ \\
\hline \multirow[t]{2}{*}{ Para (number) } & $0.250(2.063)$ & $-0.375(-1.795)$ \\
\hline & Adj $P=0.043^{*}$ & Adj $P=0.082$ \\
\hline Time since delivery & $-0.091(-0.784)$ & $0.374(2.082)$ \\
\hline ( $\geq 6$ weeks vs $<6$ weeks) & Adj $P=0.436$ & Adj $P=0.045^{*}$ \\
\hline General help-seeking & $-0.155(-1.349)$ & $0.090(0.519)$ \\
\hline (\% mean score) & Adj $P=0.182$ & Adj $P=0.607$ \\
\hline \multirow{2}{*}{$\begin{array}{l}\text { Partner support } \\
(\% \text { mean score) }\end{array}$} & $-0.238(-2.038)$ & $-0.199(-1.108)$ \\
\hline & Adj $P=0.045^{*}$ & Adj $P=0.276$ \\
\hline \multirow[t]{2}{*}{ Constant } & $(4.777)$ & $(2.896)$ \\
\hline & Adj $P<0.00 I^{*}$ & Adj $P=0.007^{*}$ \\
\hline
\end{tabular}

Notes: *Statistically significant at $P<0.05 ; t$, Student's $t$-test.

Abbreviations: $\beta$, coefficient of determination; Adj, adjusted; C-section, cesarean section. 


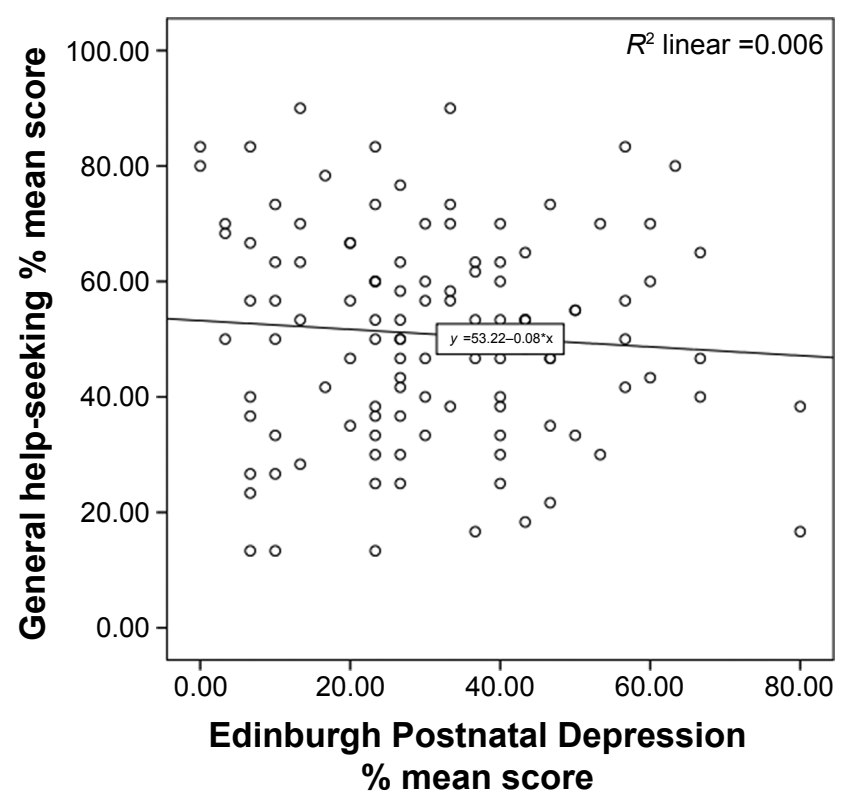

Figure I Relationship between general help-seeking score and depression score $(P=0.506)$.

that were indirectly related to depression as observed in this study (Figures 1 and 2), although GHSB was not proven to be statistically significant in this study.

Numerous studies have investigated help-seeking behavior among postpartum women. ${ }^{22-24}$ The GHS tool in this study was found to be practical and convenient as its statements cover a wide array of resources to which women can actually refer for help. For instance, postpartum Saudi women were able to acknowledge their extended family members - spouse

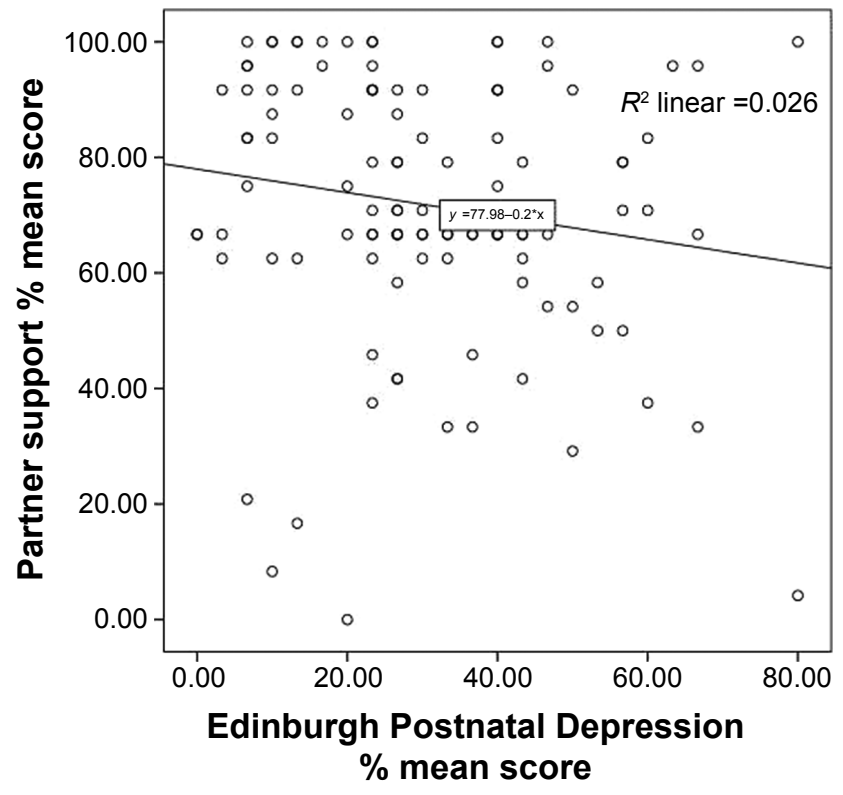

Figure 2 Relationship between partner support score and depression score $(P=0.023)$.
(54\%), parents (60\%), and relatives (42\%) - as their primary help providers, which is a cultural norm in the tribally bound Saudi community. Postpartum women in this population generally do not seek help for depression. ${ }^{25}$ Some studies actually noted rates similar to those found in studies among Hispanic/Latino women, which showed that $44.8 \%$ of the 6,672 women in their sample sought help by speaking to family members or partners. ${ }^{26}$ Although the Saudi Arabian community is considered religiously conservative, only $21 \%$ sought help from a religious figure. Apparently, women found physicians and mental health professionals more trustworthy to contact for help and advice, $24 \%$ and $32 \%$, respectively.

Younger women ( $\leq 25$ years) showed higher helpseeking scores $(55.8 \pm 19.0)$ than older women ( $\geq 36$ years) (50.8 \pm 22.0 ); however, the difference between the age groups was not statistically significant. This finding was similar to that in a study that reported the age of postpartum women was not significantly associated with their help-seeking behavior. ${ }^{27}$ Nevertheless, other studies reported that age was significantly associated with seeking professional assistance ${ }^{28}$ and that a significant positive mild-to-moderate correlation between depression score and maternal age exists. ${ }^{29}$ Younger women were slightly more reliant on their partners for support in this study, which might indicate that younger women tend to have lower self-confidence and/or less postpartum experience.

The mode of delivery was considered a key associative factor when investigating depression. Normal vaginal delivery is more frequent than $\mathrm{C}$-section deliveries, ${ }^{30}$ but each is associated with distinct pros and cons, ${ }^{31}$ making it hard to predict which has a more significant association with depression. One study proved that women who underwent normal or instrumental vaginal delivery were at lower risk of depression, when compared to the emergency $\mathrm{C}$-section group. ${ }^{32}$ Postpartum women undergoing normal vaginal delivery might endure minor-to-moderate pelvic or perennial pain, requiring sitz baths and pain relief drugs as needed. Women who undergo $\mathrm{C}$-sections endure less labor pain, but more intense, activity-restricting postpartum pain, in addition to the high risk of wound infection and body image disturbance. Normal vaginal deliveries are less costly than C-sections in this setting, and women are usually discharged earlier. Newborns delivered normally are less likely to endure neonatal complications compared to those delivered via C-sections. Also, women post C-section are not advised to conceive for 1.5 years after delivery. In this study, postpartum women who underwent normal delivery were slightly more depressed 
and sought more help compared to the C-section group, yet the $\mathrm{C}$-section group showed better perception toward their spouses as supportive partners, although the difference between the groups was not statistically significant. These results were similar to those in a study that also reported no significant difference between EPDS scores among various modes of delivery. ${ }^{31}$ Therefore, a regression model was constructed to adjust by the mode of delivery and control for other confounders.

Among the normal vaginal delivery group, higher para women were significantly more depressed. Some studies noted no significant association between PPD scores and parity or gravidity. ${ }^{31,33}$ Although higher para numbers mean that women should possess more postpartum experience, higher para women in this study had higher help-seeking scores, yet less PS. This might indicate that postpartum women with children tend to rely more on their family/parental support, but exhibit a lower perception of their supportive spouses who might be busy caring for their older children. This finding was comparable to those in a study that observed higher depression scores among multigravida women. ${ }^{34}$

An interesting finding in this study among the C-section group was their higher depression scores at $\geq 6$ weeks postpartum. A recent study in the USA has mapped depression scores over a timeline and found a significant temporal trend for depression scores decreasing until 6 months and then rebounding at 18 months. ${ }^{35}$ In the first few weeks postdelivery, Saudi women are expected to receive visits from the extended family, tribal relatives, and friends. In addition, the arrangement of feasts to celebrate the occasion is part of Saudi culture. These factors might help counteract depression, in a similar way to the traditions of other cultures. ${ }^{22}$ Furthermore, religious regulations prohibit performing sexual intercourse before 40 days postpartum ( $~ 6$ weeks). In addition, working women are expected to report for duty after finishing their maternity leave ( 2 months), requiring them to send their children to childcare centers. The increase in depression scores $\geq 6$ weeks postpartum might be related to such factors.

The findings in this study might not be generalizable to some settings, but rather to populations with comparable characteristics. The Saudi community can be considered culturally conservative and tribally bound where extended families reside near one another. Furthermore, this study focused mainly on the pregnancy-related characteristics that could be associated with PPD. However, depression could be linked to other psychological, social, financial, lifestyle stressors, or weight changes, which are beyond the scope of this study, and thus the current findings could be related to such contributing factors. In addition, there may have been a social desirability bias at work, meaning that under pressure from their conservative culture, postpartum women might have underreported depression in this study. The family might deny the fact that women with newborn babies can actually become depressed, especially given the fact they celebrate birth as a social occasion.

The researchers incorporated the GHS scale and PS scale as potential contributing and modifiable factors for PPD, an approach adding strength to the current study. This method can be adopted and used with other populations for testing the cross-cultural validation of the tool. A stronger study design would be a pre-post same-group interventional design where baseline depression is assessed prior to delivery along with a follow-up depression assessment postpartum. This would clearly identify whether PPD is related specifically to pregnancy-related factors or other demographic and social stressors.

The confidence of postpartum women can be nourished by advising their spouses and family members to support them throughout this experience. Health care providers should advise women prior to delivery to seek help postpartum whether from a family member, a friend, a health provider, or even religious scholars. The creation of postpartum social clubs under the coordination of health care institutions provides a great opportunity to counteract depression. During maternal consultations, health care workers should also watch for pregnancy-related characteristics that have been proven in this study to be significantly associated with PPD.

\section{Acknowledgment}

The authors would like to express their sincere appreciation to the study's participants without whom the project would not have been possible. The authors declare that there is no funding.

\section{Author contributions}

All authors contributed toward data analysis, drafting and critically revising the paper and agree to be accountable for all aspects of the work.

\section{Disclosure}

The authors report no conflicts of interest in this work.

\section{References}

1. Robertson E, Grace S, Wallington T, Stewart DE. Antenatal risk factors for postpartum depression: a synthesis of recent literature. Gen Hosp Psychiatry. 2004;26:89-295. 
2. Murray L, Cooper PJ. Postpartum Depression and Child Development. London: The Guilford Press; 1997.

3. Murray L, Sinclair D, Cooper P, Ducournau P, Turner P, Stein A. The socioemotional development of 5-year-old children of postnatally depressed mothers. J Child Psychol Psychiatry. 1999;40(8): 1259-1271.

4. World Health Organization. Women and Health: Today's Evidence Tomorrow's Agenda. World Health Organization; 2009. p.138.

5. Beck C. Predictors of postpartum depression: an update. Nurs Res. 2001; 50(5):275-285.

6. Dennis C-L, Ross L. Women's perceptions of partner support and conflict in the development of postpartum depressive symptoms. $J A d v$ Nurs. 2006;56(5):588-599.

7. Eberhard-Gran M, Eskild A, Tambs K, Samuelsen SO, Opjordsmoen S. Depression in postpartum and non-postpartum women: prevalence and risk factors. Acta Psychiatr Scand. 2002;106(6):426-433.

8. Zhang R, Chen Q, Li Y. Study for the factors related to postpartum depression. Zhonghua Fu Chan Ke Za Zhi. 1999;34(4):231-233.

9. Wilson CJ, Deane FP, Ciarrochi JV, Rickwood D. Measuring help seeking intentions: Properties of the General Help Seeking Questionnaire. Can J Couns. 2005;39(1):15-28.

10. D’Avanzo B, Barbato A, Erzegovesi S, Lampertico L, Rapisarda F, Valsecchi L. Formal and informal help-seeking for mental health problems. A survey of preferences of Italian students. Clin Pract Epidemiol Ment Health. 2012;8:47.

11. O'hara MW, Swain AM. Rates and risk of postpartum depression a meta-analysis. Int Rev Psychiatry. 1996;8(1):37-54.

12. Guedeney N, Fermanian J. Validation study of the French version of the Edinburgh Post Natal Depression Scale (EPDS): new results about use and psychometric properties. Eur Psychiatry. 1998;13:813-817.

13. Montazeri A, Torkan B, Omidvari S. The Edinburgh Postnatal Depression Scale (EPDS): translation and validation study of the Iranian version. BMC Psychiatry. 2007;7(1):11.

14. Al-Modayfer O, Alatiq Y, Khair O, Abdelkawi S. Postpartum depression and related risk factors among Saudi females. Int $J$ Culture Ment Health. 2015;8(3):316-324.

15. Shrestha SD, Pradhan R, Tran TD, Gualano RC, Fisher JR. Reliability and validity of the Edinburgh Postnatal Depression Scale (EPDS) for detecting perinatal common mental disorders (PCMDs) among women in low-and lower-middle-income countries: a systematic review. $B M C$ Pregnancy Childbirth. 2016;16(1):72.

16. Straughen JK, Caldwell CH, Young AA, Misra DP. Partner support in a cohort of African American families and its influence on pregnancy outcomes and prenatal health behaviors. BMC Pregnancy Childbirth. 2013;13:187.

17. IBM Corp. Released 2017. IBM SPSS Statistics for Windows, Version 23.0. Armonk, NY: IBM Corp.

18. Alasoom LI, Koura MR. Predictors of postpartum depression in the eastern province capital of Saudi Arabia. J Family Med Prim Care. 2014;3(2):146.

19. Barker ED, Copeland W, Maughan B, Jaffee SR, Uher R. Relative impact of maternal depression and associated risk factors on offspring psychopathology. Br J Psychiatry. 2012;200(2):124-129.
20. Murray L, Cooper P, Hipwell A. Mental health of parents caring for infants. Arch Womens Ment Health. 2003;6(2):s71-s77.

21. Moraes IG, Pinheiro RT, Silva RA, Horta BL, Sousa PL, Faria AD. Prevalence of postpartum depression and associated factors. Rev Saúde Pública. 2006;40(1):65-70.

22. Park VM, Goyal D, Nguyen T, Lien H, Rosidi D. Postpartum traditions, mental health, and help-seeking considerations among Vietnamese American women: a mixed-methods pilot study. J Behav Health Serv Res. Epub 2015 Aug 15:1-4.

23. Callister LC, Beckstrand RL, Corbett C. Postpartum depression and help-seeking behaviors in immigrant Hispanic women. $J$ Obstet Gynecol Neonatal Nurs. 2011;40(4):440-449.

24. McIntosh J. Postpartum depression: women's help-seeking behaviour and perceptions of cause. J Adv Nurs. 1993;18(2):178-184.

25. Liberto TL. Screening for depression and help-seeking in postpartum women during well-baby pediatric visits: an integrated review. J Pediatr Health Care. 2012;26(2):109-117.

26. Barrera AZ, Nichols AD. Depression help-seeking attitudes and behaviors among an Internet-based sample of Spanish-speaking perinatal women. Rev Panam Salud Publica. 2015;37(3):148-153.

27. Azale T, Fekadu A, Hanlon C. Treatment gap and help-seeking for postpartum depression in a rural African setting. BMC Psychiatry. 2016;16(1):1.

28. Fonseca A, Gorayeb R, Canavarro MC. Women's help-seeking behaviours for depressive symptoms during the perinatal period: sociodemographic and clinical correlates and perceived barriers to seeking professional help. Midwifery. 2015;31(12):1177-1185.

29. Ghotbi F, Akbari Sene A, Azargashb E, et al. Women's knowledge and attitude towards mode of delivery and frequency of cesarean section on mother's request in six public and private hospitals in Tehran, Iran, 2012. J Obstet Gynaecol Res. 2014;40(5):1257-1266.

30. Golbasi Z, Kelleci M, Kisacik G, Cetin A. Prevalence and correlates of depression in pregnancy among Turkish women. Matern Child Health J. 2010;14(4):485-491.

31. Leite AC, Júnior EA, Helfer TM, Marcolino LA, Vasques FA, Sá RA. Comparative analysis of perinatal outcomes among different types of deliveries in term pregnancies in a reference maternity of Southeast Brazil. Ceska gynekologie/Ceska lekarska spolecnost J Ev Purkyne. 2015;81(1):54-57.

32. Yang SN, Shen LJ, Ping T, Wang YC, Chien CW. The delivery mode and seasonal variation are associated with the development of postpartum depression. J Affect Disord. 2011;132(1):158-164.

33. Hayworth J, Little BC, Carter SB, Raptopoulos P, Priest RG, Sandler M. A predictive study of post-partum depression: some predisposing characteristics. Br J Med Psychol. 1980;53(2):161-167.

34. Ajinkya S, Jadhav PR, Srivastava NN. Depression during pregnancy: prevalence and obstetric risk factors among pregnant women attending a tertiary care hospital in Navi Mumbai. Ind Psychiatry J. 2013; 22(1):37.

35. Kothari C, Wiley J, Moe A, Liepman MR, Tareen RS, Curtis A. Maternal depression is not just a problem early on. Public Health. 2016;137: 154-161.
Neuropsychiatric Disease and Treatment

\section{Publish your work in this journal}

Neuropsychiatric Disease and Treatment is an international, peerreviewed journal of clinical therapeutics and pharmacology focusing on concise rapid reporting of clinical or pre-clinical studies on a range of neuropsychiatric and neurological disorders. This journal is indexed on PubMed Central, the 'PsycINFO' database and CAS,

\section{Dovepress}

and is the official journal of The International Neuropsychiatric Association (INA). The manuscript management system is completely online and includes a very quick and fair peer-review system, which is all easy to use. Visit http://www.dovepress.com/testimonials.php to read real quotes from published authors. 